\title{
Low frame coherence via zero-mean tensor embeddings
}

\author{
Bernhard G. Bodmann and John I. Haas IV*
}

\begin{abstract}
This paper is concerned with achieving optimal coherence for highly redundant real unit-norm frames. As the redundancy grows, the number of vectors in the frame becomes too large to admit equiangular arrangements. In this case, other geometric optimality criteria need to be identified. To this end, we use an iteration of the embedding technique by Conway, Hardin and Sloane. As a consequence of their work, a quadratic mapping embeds equiangular lines into a simplex in a real Euclidean space. Here, higher degree polynomial maps embed highly redundant unit-norm frames to simplices in high-dimensional Euclidean spaces. We focus on the lowest degree case in which the embedding is quartic.
\end{abstract}

Keywords: Grassmannian packings, tensor embeddings

\section{INTRODUCTION}

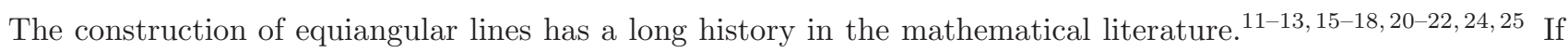
the number of unit-norm vectors spanning these lines cannot be enlarged any more without changing the set of angles/distances between them, then these vectors constitute an example of an optimal packing. Such packings have applications ranging from coding, fiber-optic or wireless communications to phase retrieval and quantum information theory $]^{3] 6}$ An analytic formulation of equiangular lines as solutions of an optimization problem is the so-called Welch bound $\frac{23}{23}$ It can be obtained by combining a mapping of Conway, Hardin and Sloane ${ }^{8}$ with a spherical cap packing bound by Rankin ?20

In an earlier work, the case of redundancy beyond the equiangular regime was addressed by combining the embedding by Conway, Hardin and Sloane with the orthoplex bound, which is saturated by the example of maximal sets of mutually unbiased bases. With the help of relative difference sets, previously unknown examples of Grassmannian packings could be constructed, $[\sqrt[5]{5}$ for examples, see the tables of Refs. $7,[1]$ for instances of Grassmannian frames with redundancies varying between that of maximal equiangular frames and maximal mutually unbiased bases.

Here, we iterate the embedding to obtain higher degree polynomial maps that are used to embed specific unit-norm frames to simplices. As a result, we identify several cases of optimal packings.

\section{PRELIMINARIES}

\subsection{Frame Theory}

Let $\left\{e_{j}\right\}_{j=1}^{m}$ denote the canonical orthonormal basis for the Hilbert space $\mathbb{R}^{m}$. A sequence of vectors $\mathcal{F}=$ $\left\{f_{j}\right\}_{j=1}^{n} \subset \mathbb{R}^{m}$ is a (finite) frame for $\mathbb{R}^{m}$ if it spans the entire Hilbert space. From now on, we reserve the symbols $m$ and $n$ to refer to the dimension of the span of a frame and the cardinality of a frame, respectively. The redundancy of a given frame is the ratio $\frac{n}{m}$.

A frame $\mathcal{F}=\left\{f_{j}\right\}_{j=1}^{n}$ is $a$-tight if

$$
\sum_{j=1}^{n} f_{j} f_{j}^{*}=a \mathbf{I}_{m}, \text { for some } a>0
$$

where $\mathbf{I}_{m}$ denotes the $m \times m$ identity matrix. The frame is unit-norm if each frame vector has norm $\left\|f_{j}\right\|=1$.

Given a unit-norm frame $\mathcal{F}=\left\{f_{j}\right\}_{j=1}^{n}$, its frame cosines are the elements of the set

$$
\Theta_{\mathcal{F}}:=\left\{\left|\left\langle f_{j}, f_{l}\right\rangle\right|: j \neq l\right\},
$$

*This work was partially supported by NSF grant DMS-1412524 and the AMS-Simons Travel grant. 
and we say that $\mathcal{F}$ is $k$-angular if $\left|\Theta_{\mathcal{F}}\right|=k$ for some $k \in \mathbb{N}$. If $\Theta_{\mathcal{F}}$ has only one element and $\mathcal{F}$ is tight, then we speak of an equiangular tight frame.

Let $\Omega_{n, m}(\mathbb{R})$ denote the space of unit-norm frames for $\mathbb{R}^{m}$ consisting of $n$ vectors. Given any set of unit vectors, $\mathcal{F}=\left\{f_{j}\right\}_{j=1}^{n} \subset \mathbb{F}^{m}$, its coherence is defined by

$$
\mu(\mathcal{F})=\max _{j \neq l}\left|\left\langle f_{j}, f_{l}\right\rangle\right|
$$

We define and denote the Grassmannian constant as

$$
\mu_{n, m}(\mathbb{R})=\min _{\mathcal{F} \in \Omega_{n, m}(\mathbb{R})} \mu(\mathcal{F}) .
$$

Correspondingly, a frame $\mathcal{F} \in \Omega_{n, m}(\mathbb{R})$ is a Grassmannian frame if

$$
\mu(\mathcal{F})=\mu_{n, m}(\mathbb{R}) .
$$

\section{ZERO-MEAN TENSOR EMBEDDINGS}

Our path toward identifying certain optimal line packings involves a two step process. First, we apply a normpreserving map to the frame vectors, thereby embedding the frame into a higher dimensional real sphere. For the second step, we interpret the embedded vectors as the centers of spherical caps (which we discuss below) and exploit the cap packing results of Rankin ${ }^{20}$ If a frame embeds into an optimal cap packing and certain additional conditions are satisfied, then the minimal coherence of the lifted frame is verified by the isometric nature of the the embedding.

We begin by defining the aforementioned family of norm-preserving maps. We denote the unit sphere in $\mathbb{R}^{m}$ by $\mathcal{S}\left(\mathbb{R}^{m}\right)$ and we let $\mathcal{B}_{\mathrm{SA}}\left(\mathbb{R}^{m}\right)$ denote the real vector space of self-adjoint linear maps on $\mathbb{R}^{m}$. From here on, $\omega$ is a random vector with values in $\mathcal{S}\left(\mathbb{R}^{m}\right)$ and $\mathbb{E}$ denotes the expectation with respect to the underlying uniform probability measure on $\mathcal{S}\left(\mathbb{R}^{m}\right)$.

DeFinition 3.1. The first zero-mean tensor embedding is defined and denoted by

$$
\mathbf{Q}_{m}^{(1)}: \mathcal{S}\left(\mathbb{R}^{m}\right) \rightarrow \mathcal{B}_{\mathrm{SA}}\left(\mathbb{R}^{m}\right): x \mapsto x \otimes x^{*}-\frac{1}{m} \mathbf{I}_{m}
$$

and for $t \in \mathbb{N}$, the $(t+1)$-th zero-mean tensor embedding, $\mathbf{Q}_{m}^{(t+1)}$, is defined recursively by

$$
\mathbf{Q}_{m}^{(t+1)}: \mathcal{S}\left(\mathbb{R}^{m}\right) \rightarrow \mathcal{B}_{\mathrm{SA}}\left(\mathbb{R}^{m}\right)^{\otimes 2^{t-1}}: x \mapsto\left(\mathbf{Q}_{m}^{(t)}(x)\right)^{\otimes 2}-\mathbb{E}\left[\left(\mathbf{Q}_{m}^{(t)}(\omega)\right)^{\otimes 2}\right]
$$

For brevity, we also refer the $t$-th zero-mean tensor embedding simply as the $t$-th embedding. The purpose of subtracting the expected value is that, just as $\mathbb{E}[\omega]=0$, the mean of the embedding vanishes,

$$
\mathbb{E}\left[\mathbf{Q}_{m}^{(t)}(\omega)\right]=0
$$

In comparison with the action of of taking simple tensor powers of $x \otimes x^{*}$, the dimension of the range of the embedding is reduced by subtracting the expectation, as we show in the next theorem. To simplify notation, for each $t \geq 2$, we write

$$
\mathbf{V}^{(t)}:=\mathbb{E}\left[\left(\mathbf{Q}_{m}^{(t-1)}(\omega)\right)^{\otimes 2}\right]
$$

Theorem 3.2. If $t \geq 2$ and $x \in \mathbb{F}^{m}$, then $\operatorname{tr}\left[\mathbf{Q}_{m}^{(t)}(x) \mathbf{V}^{(t)}\right]=0$.

Proof. We note that for any unitary $U$ on $\mathbb{R}^{m}$,

$$
U^{\otimes 2^{t-1}} \mathbf{V}^{(t)}\left(U^{*}\right)^{\otimes 2^{t-1}}=\mathbb{E}\left[\mathbf{Q}_{m}^{(t-1)}(U \omega) \otimes \mathbf{Q}_{m}^{(t-1)}(U \omega)\right]=\mathbf{V}^{(t)}
$$


because $U \omega$ and $\omega$ are identically distributed. This implies that

$$
\begin{aligned}
\operatorname{tr}\left[\mathbf{Q}_{m}^{(t-1)}(x) \otimes \mathbf{Q}_{m}^{(t-1)}(x) \mathbf{V}^{(t)}\right] & =\operatorname{tr}\left[\mathbf{Q}_{m}^{(t-1)}(x) \otimes \mathbf{Q}_{m}^{(t-1)}(x) U^{\otimes 2^{t-1}} \mathbf{V}^{(t)}\left(U^{*}\right)^{\otimes 2^{t-1}}\right] \\
& =\operatorname{tr}\left[\mathbf{Q}_{m}^{(t-1)}\left(U^{*} x\right) \otimes \mathbf{Q}_{m}^{(t-1)}\left(U^{*} x\right) \mathbf{V}^{(t)}\right]
\end{aligned}
$$

and by averaging with respect to the choice of $U^{*}$ among all unitaries,

$$
\operatorname{tr}\left[\mathbf{Q}_{m}^{(t-1)}(x) \otimes \mathbf{Q}_{m}^{(t-1)}(x) \mathbf{V}^{(t)}\right]=\operatorname{tr}\left[\left(\mathbf{V}^{(t)}\right)^{2}\right] .
$$

Consequently,

$$
\operatorname{tr}\left[\mathbf{Q}_{m}^{(t)}(x) \mathbf{V}^{(t)}\right]=\operatorname{tr}\left[\left(\mathbf{Q}_{m}^{(t-1)}(x) \otimes \mathbf{Q}_{m}^{(t-1)}(x)-\mathbf{V}^{(t)}\right) \mathbf{V}^{(t)}\right]=0
$$

口

The space of symmetric tensors in $\left(\mathbb{R}^{d}\right)^{\otimes 2}$ is of dimension $d(d+1) / 2$, and with an additional orthogonality condition the range is reduced to a subspace of dimension $d(d+1) / 2-1=(d+2)(d-1) / 2$. Iterating this dimensionality bound yields a maximal dimension of the subspace containing the range of $\mathbf{Q}_{m}^{(t)}$. Accordingly, we define and denote the first embedding dimension by

$$
\mathbf{d}_{m}^{(1)}:=\frac{(m+2)(m-1)}{2}
$$

and, for $t \in \mathbb{N}$, we define the $(t+1)$-th embedding dimension recursively by

$$
\mathbf{d}_{m}^{(t+1)}:=\frac{1}{2}\left(\mathbf{d}_{m}^{(t)}+2\right)\left(\mathbf{d}_{m}^{(t)}-1\right) .
$$

For example, the second embedding dimension is

$$
\mathbf{d}_{m}^{(2)}=\frac{\left(m^{2}+m+2\right)\left(m^{2}+m-4\right)}{8}
$$

and the third embedding dimension is

$$
\mathbf{d}_{m}^{(3)}=\frac{((m-1) m(m+1)(m+2)+8)((m-1) m(m+1)(m+2)-16)}{128} .
$$

In particular, Theorem 3.2 yields the following corollary.

COROLlary 3.3. The range of the map $\mathbf{Q}_{m}^{(t)}$ is contained in a subspace of $\mathcal{B}_{\mathrm{SA}}\left(\mathbb{R}^{m}\right)^{\otimes 2^{t-1}}$, whose dimension is at most equal to $\mathbf{d}_{m}^{(t)}$.

\section{RANKIN'S BOUND AND ACHIEVING OPTIMAL COHERENCE}

In this section, we show how to exploit Rankin's classical bounds for spherical cap packings ${ }^{20}$ and deduce the optimality properties of certain frames. Given $d \in \mathbb{N}$, a unit vector $x \in \mathcal{S}\left(\mathbb{R}^{d}\right)$ and a real number $\theta \in(0, \pi]$, we define and denote the spherical cap of angular radius $\theta$ centered at $x$ as

$$
\mathcal{C}_{x}(\theta):=\left\{y \in \mathcal{S}\left(\mathbb{R}^{d}\right):\langle x, y\rangle>\cos \theta\right\},
$$

which is alternatively referred to as a $\theta$-cap when the center is arbitrary. Rankin considered following optimization problem.

Problem 4.1. Given a fixed dimension, $d$, and a fixed angle, $\theta$, what is the largest number, $n$, of $\theta$-caps, $\left\{\mathcal{C}_{x_{j}}(\theta)\right\}_{j=1}^{n}$, that one can configure on the surface of $\mathcal{S}\left(\mathbb{R}^{d}\right)$ such that $\mathcal{C}_{x_{j}}(\theta) \cap \mathcal{C}_{x_{j^{\prime}}}(\theta)=\emptyset$ for each $j, j^{\prime} \in$ $\{1,2, \ldots, n\}$ with $j \neq j^{\prime}$. As a partial solution, Rankin reformulated Problem 4.1 in terms of its inverse 
optimization problem, providing sharp upper bounds on the caps' angular radii, completely solving the problem whenever $n \leq 2 d$. We phrase his results in terms of the inner products between the caps' centers.

THEOREM 4.2. [Rankin, [20] Given any positive integer $d$ and any set of $n$ unit vectors $\left\{v_{1}, v_{2}, \ldots, v_{n}\right\}$ in $\mathbb{R}^{d}$, then

$$
\max _{\substack{j, l \in\{1,2, \ldots, n\} \\ j \neq l}}\left\langle v_{j}, v_{l}\right\rangle \geq-\frac{1}{n-1},
$$

and if $n>d+1$, then it improves to

$$
\max _{\substack{j, l \in\{1,2, \ldots, n\} \\ j \neq l}}\left\langle v_{j}, v_{l}\right\rangle \geq 0
$$

\subsection{The first embedding}

The first embedding has been used in conjunction with Rankin's cap-packing result\$20 to characterize and construct numerous families of Grassmannian frames $4[5]$ In more detail, the vectors are mapped to a selfadjoint rank-one Hermitian and projected onto the orthogonal complement of the identity matrix. The inner product between the images of two unit vectors is a polynomial of the original inner product. As a consequence, under certain assumptions, optimal packings are equivalent to packings on a Euclidean sphere, and the Rankin bound can be applied.

TheOREM 4.3. [Conway, Hardin and Sloane, $\frac{, 8]}{]}$ If $\mathcal{F}$ is a unit-norm frame of $n$ vectors in $\mathbb{F}^{m}$ and $\left\{\mathbf{Q}_{m}^{(1)}(f)\right.$ : $f \in \mathcal{F}\}$ forms a simplex in a subspace of the real space of self-adjoint $m \times m$ matrices over $\mathbb{F}$, then $\mathcal{F}$ is a Grassmannian frame. Moreover, if $n>\mathbf{d}_{m}^{(1)}+1$, and the Hilbert-Schmidt inner product of any pair from $\left\{\mathbf{Q}_{m}^{(1)}(f): f \in \mathcal{F}\right\}$ is non-positive, then $\mathcal{F}$ is a Grassmannian frame.

Proof. It is straightforward to verify that

$$
x \mapsto \tilde{T}^{(1)}(x)=\frac{m}{m-1} \mathbf{Q}_{m}^{(1)}(x)
$$

maps the unit sphere in $\mathbb{F}^{m}$ to the unit sphere in $\mathcal{B}_{\mathrm{SA}}\left(\mathbb{R}^{m}\right)$. More generally, the inner products of frame vectors $f_{j}$ and $f_{l}$ are related by

$$
\left\langle\tilde{T}^{(1)}\left(f_{j}\right), \tilde{T}^{(1)}\left(f_{l}\right)\right\rangle_{H S}=\frac{m}{(m-1)}\left(\left|\left\langle f_{j}, f_{l}\right\rangle\right|^{2}-\frac{1}{m}\right) .
$$

Now applying Rankin's bound shows that if the Hilbert-Schmidt inner product assumes the constant value $\left\langle\tilde{T}^{(1)}\left(f_{j}\right), \tilde{T}^{(1)}\left(f_{l}\right)\right\rangle_{H S}=-\frac{1}{n-1}$ when $j \neq l$, then the maximal magnitude occurring among inner products of pairs of vectors from $\mathcal{F}$ is minimized.

Moreover, if $n>\mathbf{d}_{m}^{(1)}+1$ and the maximum $\max _{j \neq l}\left\langle\tilde{T}^{(1)}\left(f_{j}\right), \tilde{T}^{(1)}\left(f_{l}\right)\right\rangle_{H S} \leq 0$, then by Rankin's bound equality holds and the frame is Grassmannian.

Converting between the (squared) inner product of the frame vectors and the Hilbert-Schmidt inner product of the embedded vectors gives the Welch and orthoplex bounds as consequence.

COROLLARY 4.4. If $\mathcal{F}$ is a unit-norm frame of $n$ vectors in $\mathbb{F}^{m}$, then $\max _{j \neq l}\left|\left\langle f_{j}, f_{l}\right\rangle\right| \geq \sqrt{\frac{n-m}{(n-1) m}}$ and if equality holds, $\mathcal{F}$ is an equiangular tight frame. Moreover, if $n>\mathbf{d}_{m}^{(1)}+1$, then $\max _{j \neq l}\left|\left\langle f_{j}, f_{l}\right\rangle\right| \geq \frac{1}{\sqrt{m}}$ and if equality holds, $\mathcal{F}$ is a Grassmannian frame. 


\subsection{The second embedding}

For the remainder of this work, we focus on the development of the analogous machinery corresponding to the second tensor embedding. In order to provide an explicit expression for second embedding, we must compute the expectation, $\mathbb{E}\left[\left(\mathbf{Q}_{m}^{(1)}(\omega)\right)^{\otimes 2}\right]$, as given in Definition [3.1. To facilitate this, we define and denote the $t$ coherence tensor (for $\mathbb{R}^{m}$ ) by

$$
\mathbf{K}_{m}^{(t)}:=\int_{\mathcal{O}^{m}}\left(U P U^{*}\right)^{\otimes t} d \mu(U),
$$

where $\mathcal{O}_{\mathbb{R}^{m}}$ denotes the matrix group of $m \times m$ orthogonal matrices, $\mu$ denotes the unique, left-invariant Haarmeasure on $\mathcal{O}_{\mathbb{R}^{m}}$, and $P$ is any $m \times m$ orthogonal projection onto a one-dimensional subspace of $\mathbb{R}^{m}$. In the following proposition, we provide an analytic expression for the 2-coherence tensor. In order to express its dependence on the underlying field and to simplify notation, we define the constants

$$
\mathbf{a}_{m}:=\frac{\mathbf{d}_{m}^{(1)}+(m-1)^{2}}{m^{2} \mathbf{d}_{m}^{(1)}} \text { and } \mathbf{b}_{m}:=\frac{\mathbf{a}_{m}}{3},
$$

and for each $j, j^{\prime} \in\{1,2, \ldots, n\}$, we denote the canonical matrix units for $\mathbb{R}^{m \times m}$ by $E_{j, j^{\prime}}:=e_{j} \otimes\left(e_{j^{\prime}}\right)^{*}$.

Proposition 4.5. [see [19, Ch. 7-9] for details] The 2-coherence tensor for $\mathbb{R}^{m}$ can be expressed in terms of the constants $\mathbf{a}_{m}$ and $\mathbf{b}_{m}$ by

$$
\mathbf{K}_{m}^{(2)}=\mathbf{a}_{m} \sum_{j=1}^{m} E_{j, j} \otimes E_{j, j}+\mathbf{b}_{m} \sum_{\substack{j, j^{\prime}=1 \\ j \neq j^{\prime}}}^{m}\left(E_{j, j^{\prime}} \otimes E_{j^{\prime}, j}+E_{j, j} \otimes E_{j^{\prime}, j^{\prime}}+E_{j, j^{\prime}} \otimes E_{j, j^{\prime}}\right) .
$$

Proof. As defined, the 2-coherence tensor is an average of rank one orthogonal projections, yielding the trace normalization $\operatorname{tr}\left(\mathbf{K}_{m}^{(2)}\right)=1$. Its explicit form follows by the invariance properties of $\operatorname{tr}\left(\mathbf{K}_{m}^{(2)}\right)$ under the tensor representation of the orthogonal or unitary group (see [19, Ch. 7-9] for details).

In light of the explicit expression for $\mathbf{K}_{m}^{(2)}$ from Proposition 4.5, the value of its squared Hilbert Schmidt norm follows by direct computation, which we record in the following corollary.

COROLlary 4.6. The squared Hilbert-Schmidt norm of the 2-coherence tensor is

$$
\left\|\mathbf{K}_{m}^{(2)}\right\|_{H S}^{2}=\operatorname{tr}\left(\left(\mathbf{K}_{m}^{(2)}\right)^{2}\right)=m \mathbf{a}_{m}^{2}+m(m-1)\left(3 \mathbf{b}_{m}^{2}\right)=\mathbf{a}_{m}
$$

With these basic properties of the 2-coherence tensor established, next we compute $\mathbb{E}\left[\left(\mathbf{Q}_{m}^{(1)}(\omega)\right)^{\otimes 2}\right]$ and, in particular, provide the desired concrete expression of the second tensor embedding.

Proposition 4.7. We have have $\mathbf{V}^{(2)}=\mathbf{K}_{m}^{(2)}-\frac{1}{m^{2}} \mathbf{I}_{m} \otimes \mathbf{I}_{m}$; in particular, the second embedding is given by

$$
\mathbf{Q}_{m}^{(2)}: \mathcal{S}\left(\mathbb{R}^{m}\right) \rightarrow \mathcal{B}_{\mathrm{SA}}\left(\mathbb{R}^{m}\right)^{\otimes 2}: x \mapsto\left(\mathbf{Q}_{m}^{(1)}(x)\right) \otimes\left(\mathbf{Q}_{m}^{(1)}(x)\right)^{*}-\mathbf{K}_{m}^{(2)}+\frac{1}{m^{2}} \mathbf{I}_{m} \otimes \mathbf{I}_{m}
$$

Proof. Upon the expansion of $\mathbf{Q}_{m}^{(1)}(\omega)^{\otimes 2}$,

$$
\mathbf{Q}_{m}^{(1)}(\omega) \otimes \mathbf{Q}_{m}^{(1)}(\omega)=\left(\omega \otimes \omega^{*}\right)^{\otimes 2}-\frac{1}{m} \mathbf{I}_{m} \otimes \omega \otimes \omega^{*}-\frac{1}{m} \omega \otimes \omega^{*} \otimes \mathbf{I}_{m}+\frac{1}{m^{2}} \mathbf{I}_{m} \otimes \mathbf{I}_{m},
$$

computing the expectation term by term gives

$$
\mathbf{V}^{(t)}=\mathbb{E}\left[\mathbf{Q}_{m}^{(1)}(\omega) \otimes \mathbf{Q}_{m}^{(1)}(\omega)\right]=\mathbf{K}_{m}^{(2)}-\frac{2}{m^{2}} \mathbf{I}_{m} \otimes \mathbf{I}_{m}+\frac{1}{m^{2}} \mathbf{I}_{m} \otimes \mathbf{I}_{m},
$$


where we have used $\mathbb{E}\left[\omega \otimes \omega^{*}\right]=\frac{1}{m} \mathbf{I}_{m}$. Simplifying yields the claimed identity.

The orthogonality condition implied by Theorem 3.2 and Corollary 4.8 for the second tensor embedding thus reads as follows.

COROllary 4.8. For $t=2$,

$$
\left\langle\mathbf{Q}_{m}^{(2)}(x), \mathbf{V}^{(2)}\right\rangle_{H S}=\left\langle\mathbf{Q}_{m}^{(2)}(x), \mathbf{K}_{m}^{(2)}-\frac{1}{m^{2}} \mathbf{I}_{m} \otimes \mathbf{I}_{m}\right\rangle_{H S}=0
$$

In the following lemma, we compute the Hilbert Schmidt inner product between an arbitrary 1-embedded vector, $\mathbf{Q}_{m}^{(1)}(x)$, and the 2-coherence tensor, $\mathbf{K}_{m}^{(2)}$ and show that $\mathbf{Q}_{m}^{(2)}$ is indeed a norm-preserving embedding. Afterward, we show how the inner products between the embedded vectors relate to cosine set of the original frame.

Lemma 4.9. Given a unit vector $x \in \mathbb{F}^{m}$, then

(i) $\left\langle\left(\mathbf{Q}_{m}^{(1)}(x)\right)^{\otimes 2}, \mathbf{K}_{m}^{(2)}\right\rangle_{H S}=\mathbf{a}_{m}-\frac{1}{m^{2}}$

(ii) $\left\|\mathbf{Q}_{m}^{(2)}(x)\right\|_{H S}^{2}=1-\frac{2}{m}+\frac{2}{m^{2}}-\mathbf{a}_{m}$, which shows that the second embedding is norm preserving, up to a scale
factor.

Proof. We deduce from $\mathbf{Q}_{m}^{(2)}(x)=\left(\mathbf{Q}_{m}^{(1)}(x)\right)^{\otimes 2}-\mathbf{V}^{(2)}$ with $\operatorname{tr}\left[\mathbf{Q}_{m}^{(1)}(x)\right]=\operatorname{tr}\left[\mathbf{Q}_{m}^{(1)}(\omega)\right]=0$ that $\operatorname{tr}\left[\mathbf{Q}_{m}^{(2)}(x)\right]=$ 0 . Together with the orthogonality condition, $\left\langle\mathbf{Q}_{m}^{(2)}(x), \mathbf{V}^{(2)}\right\rangle_{H S}=0$, this gives

$$
\left\langle\mathbf{Q}_{m}^{(2)}(x), \mathbf{K}_{m}^{(2)}\right\rangle_{H S}=\left\langle\mathbf{Q}_{m}^{(2)}(x), \frac{1}{m^{2}} \mathbf{I}_{m} \otimes \mathbf{I}_{m}\right\rangle_{H S}=\frac{1}{m^{2}} \operatorname{tr}\left[\mathbf{Q}_{m}^{(2)}(x)\right]=0 .
$$

Expressing $\mathbf{Q}_{m}^{(2)}(x)$ in terms of the tensor power $\left(\mathbf{Q}_{m}^{(1)}(x)\right)^{\otimes 2}$ then implies

$$
\left\langle\left(\mathbf{Q}_{m}^{(1)}(x)\right)^{\otimes 2}, \mathbf{K}_{m}^{(2)}\right\rangle_{H S}-\left\langle\mathbf{K}_{m}^{(2)}-\frac{1}{m^{2}} \mathbf{I}_{m} \otimes \mathbf{I}_{m}, \mathbf{K}_{m}^{(2)}\right\rangle_{H S}=\operatorname{tr}\left[\left(\mathbf{K}_{m}^{(2)}\right)^{2}\right]-\frac{1}{m^{2}} \operatorname{tr}\left[\mathbf{K}_{m}^{(2)}\right]=\operatorname{tr}\left[\left(\mathbf{K}_{m}^{(2)}\right)^{2}\right]-\frac{1}{m^{2}} .
$$

Note that the explicit form for $\mathbf{K}_{m}^{(2)}$ in Proposition 4.5 implies the trace normalization

$$
\operatorname{tr}\left[\mathbf{K}_{m}^{(2)}\right]=m \mathbf{a}_{m}+m(m-1) \mathbf{b}_{m}=1
$$

and, from Corollary 4.6, we have the identity for the (squared) Frobenius norm

$$
\operatorname{tr}\left[\left(\mathbf{K}_{m}^{(2)}\right)^{2}\right]=\mathbf{a}_{m}
$$

We conclude

$$
\left\langle\left(\mathbf{Q}_{m}^{(1)}(x)\right)^{\otimes 2}, \mathbf{K}_{m}^{(2)}\right\rangle_{H S}=\mathbf{a}_{m}-\frac{1}{m^{2}}
$$

This shows Claim (ii).

To see the second claim, we re-express the squared Hilbert Schmidt norm of $\mathbf{Q}_{m}^{(2)}$ as an inner product, substitute $\mathbf{Q}_{m}^{(2)}$ in the left side of the inner product with its definition, and apply the orthogonality relation, which gives

$$
\left\langle\mathbf{Q}_{m}^{(2)}(x), \mathbf{Q}_{m}^{(2)}(x)\right\rangle_{H S}=\left\langle\left(\mathbf{Q}_{m}^{(1)}(x)\right)^{\otimes 2}-\mathbf{V}^{(2)}, \mathbf{Q}_{m}^{(2)}(x)\right\rangle_{H S}=\left\langle\left(\mathbf{Q}_{m}^{(1)}(x)\right)^{\otimes 2}, \mathbf{Q}_{m}^{(2)}(x)\right\rangle_{H S}
$$


Next, we replace $\mathbf{Q}_{m}^{(2)}$ with its definition in the right side of the inner product to obtain

$$
\left\langle\left(\mathbf{Q}_{m}^{(1)}(x)\right)^{\otimes 2}, \mathbf{Q}_{m}^{(2)}\right\rangle_{H S}=\left\|\mathbf{Q}_{m}^{(1)}(x)\right\|_{H S}^{4}-\left\langle\left(\mathbf{Q}_{m}^{(1)}(x)\right)^{\otimes 2}, \mathbf{K}_{m}^{(2)}-\frac{1}{m^{2}} \mathbf{I}_{m} \otimes \mathbf{I}_{m}\right\rangle_{H S} .
$$

The first term on the right-hand side simplifies to

$$
\left\|\mathbf{Q}_{m}^{(1)}(x)\right\|_{H S}^{4}=\left(\operatorname{tr}\left[\left(x \otimes x^{*}-\frac{1}{m} \mathbf{I}_{m}\right)^{2}\right]\right)^{2}=\left(\operatorname{tr}\left[\left(x \otimes x^{*}-\frac{1}{m} \mathbf{I}_{m}\right) x \otimes x^{*}\right]\right)^{2}=\left(\frac{m-1}{m}\right)^{2} .
$$

Using that $\operatorname{tr}\left(\mathbf{Q}_{m}^{(1)}\right)=0$ by definition, the second term in the right-hand side of Equation (5) resolves to

$$
\left\langle\left(\mathbf{Q}_{m}^{(1)}(x)\right)^{\otimes 2}, \mathbf{K}_{m}^{(2)}-\frac{1}{m^{2}} \mathbf{I}_{m} \otimes \mathbf{I}_{m}\right\rangle_{H S}=\left\langle\left(\mathbf{Q}_{m}^{(1)}(x)\right)^{\otimes 2}, \mathbf{K}_{m}^{(2)}\right\rangle_{H S}=\mathbf{a}_{m}-\frac{1}{m^{2}},
$$

where the second equality follows by the first claim of this lemma. Thus, Claim (iii) follows by combining the two terms.

Finally, we present the desired equation, which governs the relationship between a frame's cosine set and the corresponding set of signed angles between the higher-dimensional embedded vectors.

THEOREM 4.10. Given unit vectors $\left\{f_{j}\right\}_{j=1}^{n} \subset \mathbb{R}^{m}, m \geq 2$, there exist corresponding unit vectors $\left\{T_{j}^{(2)}\right\}_{j=1}^{n} \subset$ $\mathbb{R}^{\mathbf{d}_{m}^{(2)}}$ such that for any $j, l \in\{1,2, \ldots, n\}$, the following equation holds:

$$
\left\langle T_{j}^{(2)}, T_{l}^{(2)}\right\rangle=\frac{m^{2} \mathbf{d}_{m}^{(1)}}{\left(\mathbf{d}_{m}^{(1)}-1\right)(m-1)^{2}}\left(\left(\left|\left\langle f_{j}, f_{l}\right\rangle\right|^{2}-\frac{1}{m}\right)^{2}-\left(\mathbf{a}_{m}-\frac{1}{m^{2}}\right)\right) .
$$

Proof. Letting $\tilde{T}_{j}^{(2)}=\frac{\mathbf{Q}_{m}^{(2)}\left(f_{j}\right)}{\left\|\mathbf{Q}_{m}^{(2)}\left(f_{j}\right)\right\|_{H S}}$ and $\tilde{T}_{l}^{(2)}=\frac{\mathbf{Q}_{m}^{(2)}\left(f_{l}\right)}{\left\|\mathbf{Q}_{m}^{(2)}\left(f_{l}\right)\right\|_{H S}}$, we may isomorphically identify them with corresponding unit vectors $T_{j}^{(2)}, T_{l}^{(2)} \in \mathcal{S}\left(\mathbb{R}^{\mathrm{d}_{m}^{(2)}}\right)$. Thus, in terms of these vectors, we have

$$
\left\|T_{j}^{(2)}-T_{l}^{(2)}\right\|^{2}=2-2\left\langle T_{j}^{(2)}, T_{l}^{(2)}\right\rangle .
$$

On the other hand, we may pass back to their tensored forms to obtain

$$
\left\|T_{j}^{(2)}-T_{l}^{(2)}\right\|^{2}=\left\|\tilde{T}_{j}-\tilde{T}_{l}\right\|_{H S}^{2}=2-\frac{2}{\left\|\mathbf{Q}_{m}^{(2)}\left(f_{j}\right)\right\|_{H S}\left\|\mathbf{Q}_{m}^{(2)}\left(f_{l}\right)\right\|_{H S}}\left\langle\mathbf{Q}_{m}^{(2)}\left(f_{j}\right), \mathbf{Q}_{m}^{(2)}\left(f_{l}\right)\right\rangle_{H S},
$$

so by Claim (iii) of Lemma 4.9, Equation (8) reduces to

$$
\left\|T_{j}^{(2)}-T_{l}^{(2)}\right\|^{2}=2-\frac{2}{1-\frac{2}{m}+\frac{2}{m^{2}}-\mathbf{a}_{m}}\left\langle\mathbf{Q}_{m}^{(2)}\left(f_{j}\right), \mathbf{Q}_{m}^{(2)}\left(f_{l}\right)\right\rangle_{H S} .
$$

By inserting the expression for $\mathbf{a}_{m}$, we simplify further to

$$
\left\|T_{j}^{(2)}-T_{l}^{(2)}\right\|^{2}=2-\frac{2 m^{2} \mathbf{d}_{m}^{(1)}}{(m-1)^{2}\left(\mathbf{d}_{m}^{(1)}-1\right)}\left\langle\mathbf{Q}_{m}^{(2)}\left(f_{j}\right), \mathbf{Q}_{m}^{(2)}\left(f_{l}\right)\right\rangle_{H S} .
$$

Next, we expand $\mathbf{Q}_{m}^{(2)}\left(f_{l}\right)$ and obtain

$$
\left\langle\mathbf{Q}_{m}^{(2)}\left(f_{j}\right), \mathbf{Q}_{m}^{(2)}\left(f_{l}\right)\right\rangle_{H S}=\left\langle\mathbf{Q}_{m}^{(2)}\left(f_{j}\right),\left(\mathbf{Q}_{m}^{(1)}\left(f_{l}\right)\right)^{\otimes 2}\right\rangle_{H S}-\left\langle\left(\mathbf{Q}_{m}^{(1)}\left(f_{l}\right)\right)^{\otimes 2}, \mathbf{K}_{m}^{(2)}-\frac{1}{m^{2}} \mathbf{I}_{m} \otimes \mathbf{I}_{m}\right\rangle_{H S},
$$


By Corollary 4.8, the second additive term on the right-hand side of Equation 11 vanishes, so expanding $\mathbf{Q}_{m}^{(2)}\left(f_{j}\right)$ gives

$$
\left\langle\mathbf{Q}_{m}^{(2)}\left(f_{j}\right), \mathbf{Q}_{m}^{(2)}\left(f_{l}\right)\right\rangle_{H S}=\left\langle\left(\mathbf{Q}_{m}^{(1)}\left(f_{j}\right)\right),\left(\mathbf{Q}_{m}^{(1)}\left(f_{l}\right)\right)\right\rangle_{H S}^{2}-\left\langle\mathbf{K}_{m}^{(2)}-\frac{1}{m^{2}} \mathbf{I}_{m} \otimes \mathbf{I}_{m},\left(\mathbf{Q}_{m}^{(1)}\left(f_{l}\right)\right)^{\otimes 2}\right\rangle_{H S} .
$$

Because $\operatorname{tr}\left(\mathbf{Q}_{m}^{(1)}\right)=0$, the second additive term on the right-hand side of Equation (12) simplifies to

$$
\left\langle\mathbf{K}_{m}^{(2)}-\frac{1}{m^{2}} \mathbf{I}_{m} \otimes \mathbf{I}_{m},\left(\mathbf{Q}_{m}^{(1)}\left(f_{l}\right)\right)^{\otimes 2}\right\rangle_{H S}=\left\langle\mathbf{K}_{m}^{(2)},\left(\mathbf{Q}_{m}^{(1)}\left(f_{l}\right)\right)^{\otimes 2}\right\rangle_{H S}=\mathbf{a}_{m}-\frac{1}{m^{2}},
$$

where the second equality follows from the first claim of Lemma 4.9. Expanding $\mathbf{Q}_{m}^{(1)}\left(f_{j}\right)$ and $\mathbf{Q}_{m}^{(1)}\left(f_{l}\right)$ within the square of the first additive term on the right-hand side of Equation (12) yields

$$
\left\langle\left(\mathbf{Q}_{m}^{(1)}\left(f_{j}\right)\right),\left(\mathbf{Q}_{m}^{(1)}\left(f_{l}\right)\right)\right\rangle_{H S}^{2}=\left(\left|\left\langle f_{j}, f_{l}\right\rangle\right|^{2}-\frac{1}{m}\right)^{2},
$$

so substituting Equation (14) and Equation (13) into Equation (12) gives

$$
\left\langle\mathbf{Q}_{m}^{(2)}\left(f_{j}\right), \mathbf{Q}_{m}^{(2)}\left(f_{l}\right)\right\rangle_{H S}=\left(\left|\left\langle f_{j}, f_{l}\right\rangle\right|^{2}-\frac{1}{m}\right)^{2}-\left(\mathbf{a}_{m}-\frac{1}{m^{2}}\right) .
$$

The claim follows by equating Equation (7) with Equation (10), replacing the inner product with the value given in Equation (15), and then rearranging to the desired form.

We re-express this governing equation from Theorem 4.10 in terms of the ambient dimension, $m$.

Corollary 4.11. Given unit vectors $\left\{f_{j}\right\}_{j=1}^{n} \subset \mathbb{R}^{m}$, there exist corresponding unit vectors $\left\{T_{j}^{(2)}\right\}_{j=1}^{n} \subset \mathbb{R}^{\mathbf{d}_{m}^{(2)}}$ such that for any $j, l \in\{1,2, \ldots, n\}$, the following equation holds:

$$
\left\langle T_{j}^{(2)}, T_{l}^{(2)}\right\rangle=\frac{m^{2}(m+2)}{m^{3}-5 m+4}\left(\left(\left|\left\langle f_{j}, f_{l}\right\rangle\right|^{2}-\frac{1}{m}\right)^{2}-\frac{2(m-1)}{m^{2}(m+2)}\right) .
$$

We conclude by combining this embedding with Rankin's bound in order to characterize Grassmannian frames.

THEOREM 4.12. Given a frame $\mathcal{F}$ of $n$ vectors in $\mathbb{R}^{m}$, with $m \geq 2, \frac{n-\mathbf{d}_{m}^{(1)}}{n-1} \geq \frac{\mathbf{d}_{m}^{(1)}}{(m-1)^{2}}$, and $\left\{\mathbf{Q}_{m}^{(2)}(f): f \in \mathcal{F}\right\}$ forms a simplex in a subspace of $\mathcal{B}_{\mathrm{SA}}\left(\mathbb{R}^{m}\right)^{\otimes 2}$, then $\mathcal{F}$ is Grassmannian. Moreover, if $n>\mathbf{d}_{m}^{(2)}+1$ and $(m-$ $1)^{2} \geq \mathbf{d}_{m}^{(1)}$, and the inner products between pairs of $\left\{\mathbf{Q}_{m}^{(2)}(f): f \in \mathcal{F}\right\}$ are non-positive, then the frame is Grassmannian.

Proof. If $\frac{n-\mathbf{d}_{m}^{(1)}}{n-1} \geq \frac{\mathbf{d}_{m}^{(1)}}{(m-1)^{2}}$, then by estimating $\mathbf{d}_{m}^{(1)}>(m-1)^{2} / 2$, we have $n>2 \mathbf{d}_{m}^{(1)}-1$ and with $\mathbf{d}_{m}^{(1)} \geq 2$, we get $n>\mathbf{d}_{m}^{(1)}+1$, so the orthoplex bound holds, $\max _{j \neq l}\left|\left\langle f_{j}, f_{l}\right\rangle\right|^{2} \geq \frac{1}{m}$. Because the polynomial $p(x)=$ $\frac{m^{2} \mathbf{d}_{m}^{(1)}}{\left(\mathbf{d}_{m}^{(1)}-1\right)(m-1)^{2}}\left(\left(x-\frac{1}{m}\right)^{2}-\left(\mathbf{a}_{m}-\frac{1}{m^{2}}\right)\right)$ is decreasing on $\left[0, \frac{1}{m}\right]$ and increasing on $\left[\frac{1}{m}, \infty\right)$, it follows that

$$
\max _{j \neq l} p\left(\left|\left\langle f_{j}, f_{l}\right\rangle\right|^{2}\right) \leq \max \left\{p(0), p\left(\max _{j \neq l}\left|\left\langle f_{j}, f_{l}\right\rangle\right|^{2}\right)\right\} .
$$

If $\frac{n-\mathbf{d}_{m}^{(1)}}{n-1} \geq \frac{\mathbf{d}_{m}^{(1)}}{(m-1)^{2}}$, then $p(0) \leq-\frac{1}{n-1}$, and Rankin's bound implies that

$$
p\left(\max _{j \neq l}\left|\left\langle f_{j}, f_{l}\right\rangle\right|^{2}\right) \geq-\frac{1}{n-1} .
$$


Hence, if $\left\{\mathbf{Q}_{m}^{(2)}(f): f \in \mathcal{F}\right\}$ forms a simplex then equality is achieved and the frame is Grassmannian.

We continue with the more restrictive assumption $n>\mathbf{d}_{m}^{(2)}+1$, where we know Rankin's strengthened bound holds. In this case, assuming $(m-1)^{2} \geq \mathbf{d}_{m}^{(1)}$ implies $p(0) \leq 0$, which, by Rankin's bound, implies $p\left(\max _{j \neq l}\left|\left\langle f_{j}, f_{l}\right\rangle\right|^{2}\right) \geq 0$ and if all the Hilbert-Schmidt inner products between pairs of $\left\{\mathbf{Q}_{m}^{(2)}(f): f \in \mathcal{F}\right\}$ are non-positive, then equality holds and the frame is Grassmannian.

\section{EXAMPLES OF HIGH REDUNDANCY FRAMES WITH LOW COHERENCE ARISING FROM THE SECOND EMBEDDING}

To conclude this work, we present examples of frames with low coherence that arise from second tensor embedding and discuss their interesting structural properties.

EXAMPLE 5.1. A set of orthonormal bases, $\left\{\mathcal{B}_{j}\right\}_{j=1}^{k}$ for $\mathbb{R}^{m}$ are said to be mutually unbiased if $|\langle x, y\rangle|^{2}=\frac{1}{m}$ for every $x \in \mathcal{B}_{j}, y \in \mathcal{B}_{l}$ with $j \neq l$. The existence of three such bases in $\mathbb{R}^{4}$ is well-known $4[5]$ and it is also known that their union is a Grassmannian frame, $4,5,8$ in accordance with the conditions of Theorem 4.3. Out of curiousity, we fed this system of vectors through equation from Corollary 4.11 and discovered that the embedded bases, $\left\{\mathcal{B}_{j}^{(2)}\right\}_{j=1}^{3}$, have the following peculiar property.

Given any choice of $j \in\{1,2,3\}$ and any vector $T_{j}^{(2)} \in \mathcal{B}_{j}^{(2)}$, we observe that orthogonal vectors remain orthogonal when embedded and

$$
\left\{\left\langle T_{j}^{(2)}, T_{l}^{(2)}\right\rangle: T_{l}^{(2)} \in \mathcal{B}_{l}^{(2)}, l \in\{1,2,3\}, l \neq j\right\}=\left\{-\frac{1}{8}\right\}
$$

so that each embedded vector resembles the vertex of a 9-simplex relative to the eight vectors coming from the other two embedded bases.

EXAMPLE 5.2 (16 VeCtORS IN $\left.\mathbb{R}^{3}\right)$. Assisted by Sloane's database of putatively optimal packings $\frac{1}{1}$ we confirmed numerically that the second embedding maps Sloane's example of 16 vectors in $\mathbb{R}^{3}$ into a packing of 16 $\frac{\pi}{2}$-caps. After a helpful discussion with Dustin Mixon, we then ascertained that this example corresponds to the 16 lines passing through the antipodal vertices of a biscribed pentakis dodecahedron. Analytic coordinate representations of the 32 vertices of this polytope can be found at Visual Polyhedra $\stackrel{2}{2}$ an online database of various exotic polyhedra in $\mathbb{R}^{3}$. After discarding antipodal points, the remaining 16 vertices correpond to a 6 -angular unit norm tight frame, $\mathcal{F}$, for $\mathbb{R}^{3}$ with cosine set

$$
\Theta_{\mathcal{F}}=\left\{\sqrt{\frac{1}{15}(5-2 \sqrt{5})}, \frac{1}{\sqrt{5}}, \frac{1}{3}, \sqrt{\frac{7}{15}}, \frac{\sqrt{5}}{3}, \sqrt{\frac{1}{15}(5+2 \sqrt{5})}\right\} .
$$

Passing these values through the equation from Corollary 4.11 shows that the 16 frame vectors of $\mathcal{F}$ map to 16 vectors, $\mathcal{T}=\left\{T_{j}\right\}_{j=1}^{16}$, on the sphere in $\mathbb{R}^{14}$ for whose (signed) cosine set is

$$
\left\{\left\langle T_{j}^{(2)}, T_{l}^{(2)}\right\rangle: j \neq l\right\}=\{-1 / 5,-1 / 9,0\},
$$

meaning the embedded vectors form the centers of an optimal cap-packing according to the second of Rankin's conditions in Theorem 4.2. Unfortunately, the parameters of this example do not satisfy the sufficiency conditions from Theorem 4.12. so we may not state with certainty this is indeed a Grassmannian frame; however, curiously, we have also observed that the first embedding maps this frame into a set of vectors, $\left\{T_{j}^{(1)}\right\}_{j=1}^{16} \subset \mathbb{R}^{5}$, which forms a tight Grassmannian frame, characterized by the original orthoplex bound. The optimal incoherence of this intermediate frame has recently been observed by Fickus, Jasper, and Mixon!10 Given the evidence, we find it reasonable to posit that $\mathcal{F}$ is likely a Grassmannian frame. If so, then it would follow that

$$
\mu_{16,3}(\mathbb{R})=\sqrt{\frac{1}{15}(5+2 \sqrt{5})} .
$$


EXAMPLE 5.3 (120 VECTORS IN $\left.\mathbb{R}^{8}\right)$. The optimal coherence of this example has been verified via the Levenschtein bound,14 but we recertify it here in terms of the second tensor embedding. After discarding antipodal vectors from the 240 shortest vectors of the E8 lattice, the remaining 120 vectors form a unit norm frame, $\mathcal{F}$, for $\mathbb{R}^{8}$ with cosine set,

$$
\Theta_{\mathcal{F}}=\{0,1 / 2\} .
$$

We verify the assumptions of Theorem 4.12: $\mathbf{d}_{8}^{(1)}=35$, so $\frac{120-\mathbf{d}_{8}^{(1)}}{119}=\frac{85}{119} \geq \frac{35}{49}=\frac{\mathbf{d}_{8}^{(1)}}{(8-1)^{2}}$. One can verify that the second tensor embedding maps this frame to a regular 119-simplex, meaning the embedded vectors correspond to an optimal cap packing according to the first of Rankin's conditions from Theorem 4.2, thereby verifying that $\mathcal{F}$ is a Grassmannian frame and

$$
\mu_{120,8}(\mathbb{R})=\frac{1}{2} .
$$

\section{REFERENCES}

[1] Sloane's online tables. http://neilsloane.com/grass/

[2] Visual polyhedra. http://dmccooey.com/polyhedra/

[3] D. M. Appleby. Symmetric informationally complete-positive operator valued measures and the extended Clifford group. J. Math. Phys., 46(5):052107, 1-29, 2005.

[4] D. M. Appleby. SIC-POVMS and MUBS: geometrical relationships in prime dimension. In Foundations of probability and physics - 5, volume 1101 of AIP Conf. Proc., pages 223-232. Amer. Inst. Phys., New York, 2009.

[5] Bernhard G. Bodmann and John Haas. Achieving the orthoplex bound and constructing weighted complex projective 2-designs with Singer sets. Linear Algebra Appl., 511:54-71, 2016.

[6] B.G. Bodmann, P.G. Casazza, D. Edidin, and R. Balan. Frames for linear reconstruction without phase. In Information Sciences and Systems, 2008. CISS 2008. 42nd Annual Conference on, pages 721-726, March 2008.

[7] P. G. Casazza, A. Farzannia, J. I. Haas, and T. T. Tran. Toward the Classification of Biangular Harmonic Frames. ArXiv e-prints, October 2016.

[8] J. H. Conway, R. H. Hardin, and N. J. A. Sloane. Packing lines, planes, etc.: packings in Grassmannian spaces. Experiment. Math., 5(2):139-159, 1996.

[9] Ingrid Daubechies, A. Grossmann, and Y. Meyer. Painless nonorthogonal expansions. J. Math. Phys., 27(5):1271-1283, 1986.

[10] M. Fickus, J. Jasper, and D. G. Mixon. Packings in real projective spaces. ArXiv e-prints, July 2017.

[11] Matthew Fickus and Dustin G. Mixon. Tables of the existence of equiangular tight frames. arXiv e-print, arXiv:1504.00253, 042015.

[12] Matthew Fickus, Dustin G. Mixon, and Janet C. Tremain. Steiner equiangular tight frames. Linear Algebra Appl., 436(5):1014-1027, 2012.

[13] J. Haantjes. Equilateral point-sets in elliptic two- and three-dimensional spaces. Nieuw Arch. Wiskunde (2), 22:355-362, 1948.

[14] J.I . Haas, N. Hammen, D.G. Mixon Optimal packings of many lines in preparation

[15] T. R. Hoffman and J. P. Solazzo. Complex equiangular tight frames and erasures. Linear Algebra Appl., 437(2):549-558, 2012.

[16] R. B. Holmes and V. I. Paulsen. Optimal frames for erasures. Linear Algebra Appl., 377:31-51, 2004.

[17] John Jasper, Dustin G. Mixon, and Matthew Fickus. Kirkman equiangular tight frames and codes. IEEE Trans. Inform. Theory, 60(1):170-181, 2014.

[18] P. W. H. Lemmens and J. J. Seidel. Equiangular lines. J. Algebra, 24:494-512, 1973.

[19] Z.-Q. Ma. Group theory for physicists. World Scientific Publishing Co. Pte. Ltd., Hackensack, NJ, 2007.

[20] R. A. Rankin. The closest packing of spherical caps in $n$ dimensions. Proc. Glasgow Math. Assoc., 2:139-144, 1955. 
[21] T. Strohmer and R. W. Heath, Jr. Grassmannian frames with applications to coding and communication. Appl. Comput. Harmon. Anal., 14(3):257-275, 2003.

[22] J. H. van Lint and J. J. Seidel. Equilateral point sets in elliptic geometry. Indag. Math., 28:335-348, 1966.

[23] L. R. Welch. Lower bounds on the maximum cross correlation of signals. IEEE Trans. on Information Theory, 20(3):397-9, May 1974.

[24] P. Xia, S. Zhou, and G. B. Giannakis. Achieving the Welch bound with difference sets. IEEE Trans. Inform. Theory, 51(5):1900-1907, 2005.

[25] G. Zauner. Quantendesigns - Grundzüge einer nichtkommutativen Designtheorie. University Wien (Austria), 1999. Dissertation (Ph.D.), English translation in International Journal of Quantum Information (IJQI) 9 (1), 445-507, 2011. 